\title{
The Immediate Effect of COVID-19 Vaccination on Anticoagulation Control in Patients Using Vitamin K Antagonists
}

Chantal Visser ${ }^{1}(1)$ Joseph S. Biedermann ${ }^{1}$ Melchior C. Nierman ${ }^{2}$ Felix J.M. van der Meer ${ }^{3}$ Anouk J.W. Gulpen ${ }^{4}$ Yvonne C.F. Moors ${ }^{4}$ Suzanne C. Cannegieter ${ }^{3,5}$ Willem M. Lijfering ${ }^{5,6}$ Marieke J.H.A. Kruip ${ }^{1,7}$ on behalf of the Dutch COVID \& Thrombosis Coalition

${ }^{1}$ Department of Hematology, Erasmus MC, Erasmus University Medical Center Rotterdam, Rotterdam, The Netherlands

2 Department of Thrombosis and Anticoagulation, Atalmedial Medical Diagnostics Centers, Amsterdam, The Netherlands

${ }^{3}$ Department of Internal Medicine, Section of Thrombosis and Hemostasis, Leiden University Medical Centre, Leiden, The Netherlands

${ }^{4}$ Department of Internal Medicine, Elkerliek Hospital, Helmond, The Netherlands

${ }^{5}$ Department of Clinical Epidemiology, Leiden University Medical Center, Leiden, The Netherlands

\section{Abstract}

Keywords

- acenocoumarol

- phenprocoumon

- BNT162b2 vaccine

- COVID-19 vaccines

- anticoagulants
Background In January 2021, the Dutch vaccination program against severe acute respiratory syndrome coronavirus 2 (SARS-CoV-2) was started. Clinical studies have shown that systemic reactions occur in up to $50 \%$ of vaccine recipients. Therefore, COVID-19 vaccination could affect anticoagulation control, potentially leading to an increased risk of thrombotic events and bleeding complications.

Aims This article investigates whether the BNT162b2 vaccine affects anticoagulation control in outpatients using vitamin $\mathrm{K}$ antagonists (VKAs).

Methods A case-crossover study was performed in a cohort of outpatient VKA users from four Dutch anticoagulation clinics who received a BNT162b2 vaccine. International normalized ratio (INR) results and VKA dosages before the first vaccination, the reference period, were compared with those after the first and second vaccination.

Results A total of 3,148 outpatient VKA users were included, with a mean age (standard deviation) of 86.7 (8.7) years, of whom $43.8 \%$ were male, $67.0 \%$ used acenocoumarol, and $33.0 \%$ phenprocoumon. We observed a decrease of $8.9 \%$ of INRs within range in the standard intensity group (target INR 2.0-3.0). There was both an increased risk of supratherapeutic (odds ratio $[\mathrm{OR}]=1.34$ [95\% confidence interval $[\mathrm{Cl}] 1.08-1.67]$ ) and subtherapeutic levels $(\mathrm{OR}=1.40[95 \% \mathrm{Cl} 1.08-1.83])$ after first vaccination. In the high-intensity group (target INR 2.5-3.5), the risk of a supratherapeutic INR was 2.3 times higher after first vaccination $(\mathrm{OR}=2.29[95 \% \mathrm{Cl} 1.22-4.28])$ and 3.3 times higher after second vaccination (OR $=3.25[95 \% \mathrm{Cl} 1.06-9.97])$.

Conclusion BNT162b2 was associated with an immediate negative effect on anticoagulation control in patients treated with VKAs, so it is advisable to monitor the INR shortly after vaccination, even in stable patients. received

July 16, 2021

accepted after revision

December 6, 2021
DOI https://doi.org/

$10.1055 / \mathrm{s}-0042-1742628$

ISSN 0340-6245. (c) 2022. The Author(s).

This is an open access article published by Thieme under the terms of the Creative Commons Attribution License, permitting unrestricted use, distribution, and reproduction so long as the original work is properly cited. (https://creativecommons.org/licenses/by/4.0/)

Georg Thieme Verlag KG, Rüdigerstraße 14, 70469 Stuttgart, Germany 


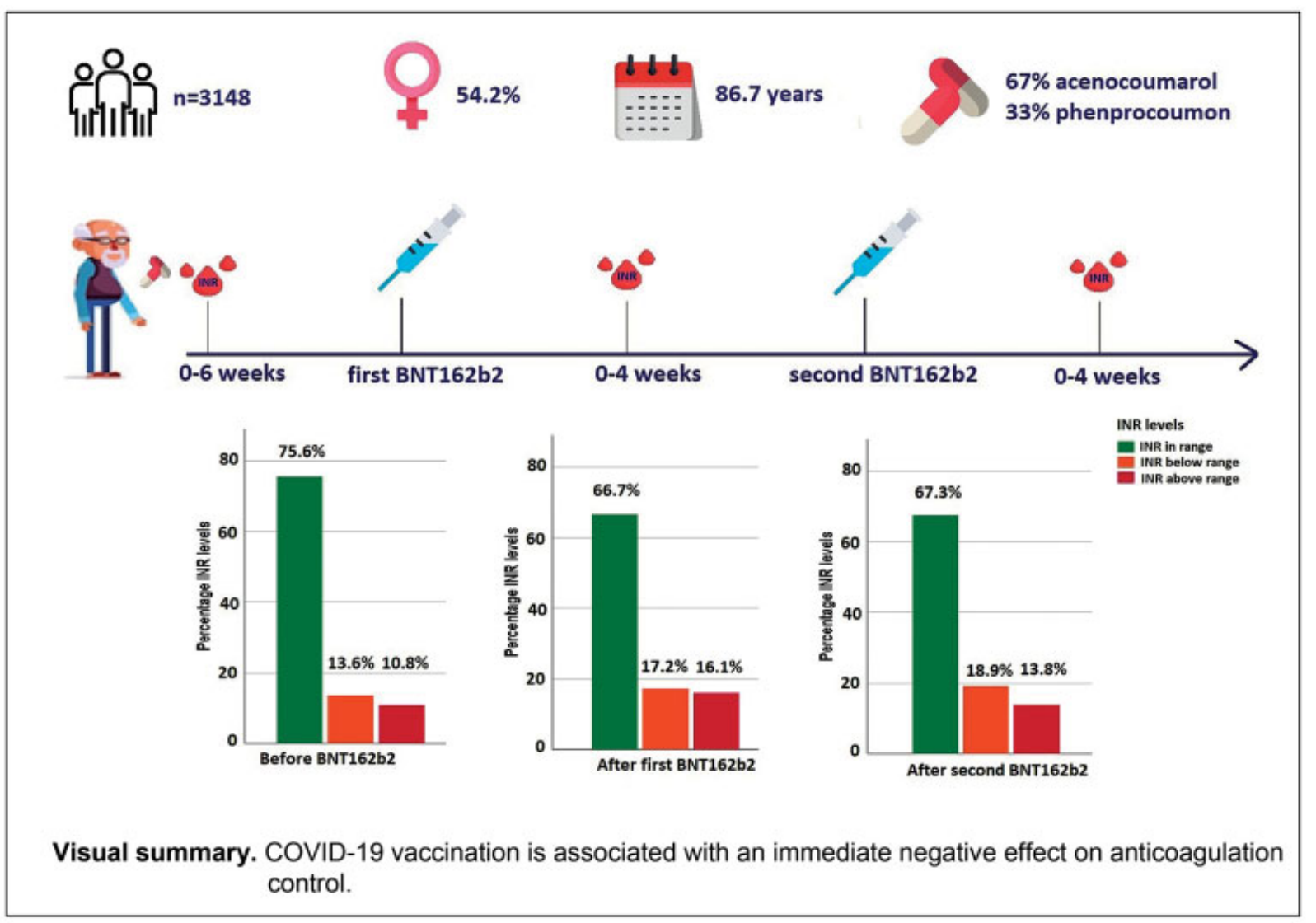

\section{Introduction}

The novel coronavirus infection disease (COVID-19), first identified in December 2019 in Wuhan, ${ }^{1}$ China, has contributed to significant morbidity and mortality worldwide, with the number of new cases still increasing. ${ }^{2}$ As of the first of July 2021, almost 200 million individuals worldwide have tested positive for severe acute respiratory syndrome coronavirus 2 (SARS-CoV-2). ${ }^{2}$ The virus can lead to various disease states, from a mild flu-like illness to very severe pneumonia with profound hypoxemia requiring mechanical ventilation. ${ }^{3,4}$ In addition to primarily affecting the respiratory system, several studies have reported effects of SARS-CoV-2 on coagulation and the cardiovascular system. ${ }^{5-8}$ COVID-19 infection has been associated with elevated D-dimers, high fibrinogen levels, and slightly prolonged prothrombin time. ${ }^{5}$ This coagulopathy is one of the most distinct prognostic factors of poor outcome in patients with COVID-19,8 and it is associated with both arterial and venous thrombotic events. ${ }^{9}$

COVID-19 coagulopathy could potentially affect the therapeutic stability in patients treated with vitamin $\mathrm{K}$ antagonists (VKAs). Treatment with VKA poses various difficulties because of their pharmacological properties. These properties include a slow onset of action and numerous interactions with dietary intake and medication. ${ }^{10}$ For instance, medications affecting albumin binding or cytochrome 450 isoenzymes, as well as dietary vitamin $\mathrm{K}$, can offset the effect of
VKAs. ${ }^{11}$ Regular measurements of the international normalized ratio (INR) are required to monitor the anticoagulant effect. Another problem is the narrow therapeutic range of VKA. Any deviation can have potentially deleterious effects, such as thrombotic events or bleeding complications. These events can be prevented by correctly dosing the INR inside the therapeutic range. ${ }^{12}$

Any systemic event such as illness, fever, or physical stress can influence a patient's INR, hence contributing to a higher risk of major events, ${ }^{13}$ as was recently shown in VKA users infected with SARS-CoV-2. ${ }^{14}$ So COVID-19 could potentially lead, also indirectly, to an increased risk of thrombotic events, bleeding, or death in patients treated with VKAs. In January 2021, the Dutch vaccination program against SARSCoV-2 was started, mainly using BNT162 b2 ${ }^{15}$ (Pfizer/BioNTech) vaccine in the elderly and people with underlying medical conditions, including many VKA users.

COVID-19 vaccination can also potentially affect anticoagulation control by directly or indirectly influencing the INR and thereby decreasing the therapeutic stability due to the abovementioned effects of SARS-CoV-2 on coagulation. Besides, clinical studies have shown that systemic reactions, including fever and chills, occur in up to $50 \%$ of vaccine recipients, depending on the type of vaccine used. ${ }^{15-17}$ It is known that systemic reactions such as fever can alter the therapeutic stability in VKA users. ${ }^{13,18}$ Therefore, we aim to investigate whether the BNT162b2 vaccine affects 
anticoagulation control in patients using VKAs. To this end, we have performed a case-crossover study in a cohort of VKA users from four anticoagulation clinics in the Netherlands.

\section{Methods}

\section{Study Design}

In this case-crossover study, we included all adult outpatient VKA users treated by four Dutch anticoagulation clinics, namely Atalmedial, trombosedienst Leiden, Star-shl, and Elkerliek trombosedienst, who received a BNT162b2 vaccine. We included outpatient VKA users who received at least one vaccine between January 1 and February 14, 2021. VKA users were excluded when 3 months before until the end of the study they (1) had been hospitalized or had received a surgical intervention, (2) started or stopped any medication interacting with VKA, (3) had a deviant INR range (e.g., 3.04.0 or $1.5-2.0$ ), or (4) switched from acenocoumarol to phenprocoumon, or vice versa (-Fig. 1). The national list of medication interacting with VKA established by the Federation of Dutch anticoagulation clinics ${ }^{19}$ was used to identify any interacting medication.

The Erasmus University Medical Centre's ethics committee granted a waiver for informed consent because of the study's retrospective nature.

\section{Data Collection}

We retrieved data from electronic patient files including baseline characteristics, year of VKA initiation, indication for
VKA treatment, INR target range and INR results, and VKA dosages. Other collected data were surgical interventions, hospital admissions, registered complications, and medication.

At the abovementioned anticoagulation clinics, all VKA users are strictly monitored at least once every 6 weeks. During each patient visit, changes in comedication, bleeding events, scheduled surgical interventions, hospital admissions, and onset of comorbidities are documented, along with the date and type of the received vaccination. The anticoagulation clinics were encouraged to measure the INR within 2 weeks after vaccination.

\section{Outcome Measures}

Our main outcome was the percentage (\%) of sub- and supratherapeutic INR after both vaccinations. We used the most recent INR measured prior to vaccination and the first INRs measured after both vaccinations. The VKA users were divided into a standard (therapeutic INR range 2.0-3.0) or high-intensity (therapeutic INR range 2.5-3.5) treatment group. For both groups, the percentage of INR results below, within, or above therapeutic range were determined prior to vaccination and after both vaccinations. We used the percentage (\%) of INRs $\geq 5$ as a surrogate marker for bleeding complications because of the heterogeneity between anticoagulation clinics in registering complications. An INR $\geq 5$ is associated with a higher risk of bleeding complications ${ }^{20}$ and will function as a surrogate marker for bleeding complications. The percentage of INRs $\geq 5$ prior to vaccination and after the first and the second vaccination was compared.

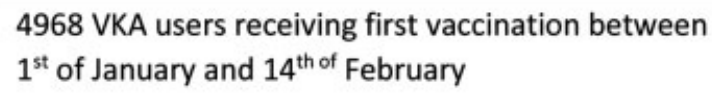

1366 VKA users who started or stopped interacting medication $\geq 2$ months prior the $1^{\text {st }}$ vaccination

\section{VKA users without interacting medication}

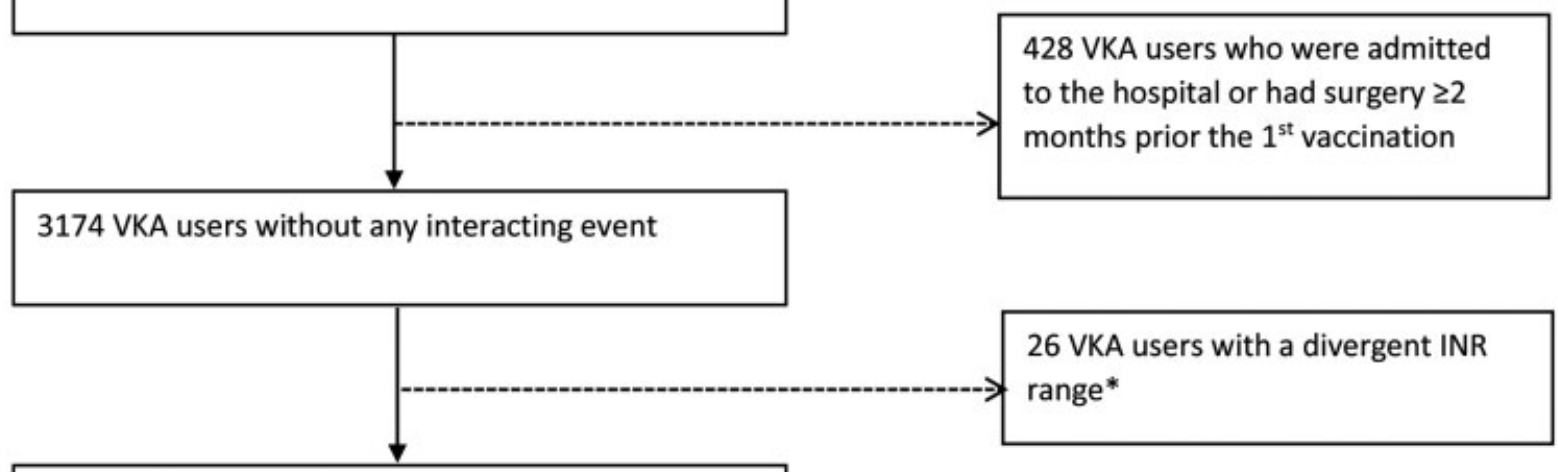

3148 VKA users eligible for the study

Fig. 1 Flow diagram of eligible vitamin $\mathrm{K}$ antagonist (VKA) users. *A divergent international normalized ratio (INR) range is defined as any therapeutic target range, which differs from 2.0 to 3.0 and 2.5 to 3.5. 
Finally, we studied the effect of both the first and second vaccination on the mean INR and VKA dosage and the percentage of INR results followed by a significant dose adjustment. A significant dose adjustment was defined as any dose adjustment of $10 \%$ or more.

\section{Statistical Analysis}

Data for continuous variables were expressed as means with standard deviation (SD) or median with interquartile range depending on the normality of the distribution. We expressed categorical data as numbers with percentages. The reference categories in all analyses were the INR and VKA dosage at the last known date before vaccination. In this study, VKA users were compared with themselves (crossover analysis). We compared absolute differences in INR and VKA dosage using paired $t$-test or a Wilcoxon signed-rank test in case of a normal distribution or skewed distribution, respectively. Percentages were compared using McNemar's test. Conditional logistic regression was used to calculate odds ratios (ORs). Subgroup analyses were performed restricting to patients with an INR within range, patients with a measurement $\leq 14$ days after vaccination, and VKA users with a poor "time in therapeutic range" (TTR), defined as a TTR $<60 \%$. The TTR was calculated using the Rosendaal method. ${ }^{21}$ To verify our results, we performed several sensitivity analyses. First, we replaced the most recent INR before vaccination with the second INR before vaccination as baseline. Second, we replaced the most recent INR with the INR measured 1 to 2 months before vaccination as baseline. If this INR was the second INR before vaccination, we included the INR before the second INR. The most recent INR before vaccination as baseline could be influenced as professionals might wait for the optimal INR to vaccination. We also selected the $20 \%$ most stable patients based on the TTR of the previous 6 months. We excluded the most recent INR before vaccination as this could potentially influence the TTR. Furthermore, we stratified by type of VKA (acenocoumarol or phenprocoumon), because phenprocoumon is associated with better anticoagulation control. ${ }^{22}$ Finally, we stratified by therapeutic range, as anticoagulation control is higher in the standard intensity group than in the high-intensity group. ${ }^{23}$ All statistical analyses were performed with IBM SPSS statistics version 25 .

\section{Results}

In total, 4,995 outpatient VKA users received their first BNT162b2 vaccine during the study period. After the exclusion criteria were applied, 3,148 outpatients were included (-Fig. 1).

Of these 3,148 patients, the mean age (SD) was 86.7 (8.7) years. Note that $43.8 \%$ were male, $67.0 \%$ used acenocoumarol, $33.0 \%$ used phenprocoumon, and $8.8 \%$ had an INR target range between 2.5 and 3.5. - Table 1 shows the patient characteristics at baseline by VKA type. Phenprocoumon users were significantly younger than the acenocoumarol users (mean age [SD] 85.9 [9.1] vs. 87.1 [8.4], $p<0.001$ ). All other clinical characteristics were similar between both groups.
In total, 1,134 VKA users completed the vaccination program. This group differed in treatment indication, age, gender, and the percentage of acenocoumarol users compared with the group receiving only one vaccination (-Supplementary Table s2, available in the online version).

\section{Anticoagulation Control in all Patients after the First Vaccination}

In the standard intensity group (INR 2.0-3.0) there was a decrease of $8.9 \%$ in INRs within range after first vaccination (-Table 2), due to a significant increase of both supratherapeutic INRs (INR $>3.0$ ) as well as subtherapeutic INRs $(<2.0)$. There was both an increased risk of supratherapeutic INR levels $(\mathrm{OR}=1.34$ [95\% confidence interval [CI] 1.08-1.67], $p=0.008$ ) and subtherapeutic levels (OR $=1.40$ [95\% CI 1.08-1.83], $p=0.012$ ) after first vaccination (-Table 3 ).

In the high-intensity group (INR 2.5-3.5), VKA users were also more likely to have an INR above range after the first vaccination (-Table 2 ). The risk of a supratherapeutic INR was 3.5 times higher after first vaccination (OR 3.50 [95\% CI 1.15-10.63], $p=0.027$ ). A subtherapeutic INR after vaccination was as often observed as prior to vaccination.

Overall, the mean INR was significantly higher after the first vaccination than before vaccination (mean INR [SD] before vs. after, 2.50 [0.57] vs. 2.54 [0.68], $p=0.001$ ) receiving at least one vaccination. The percentage of INRs $\geq 5$ prior to vaccination was similar to the percentage after vaccination. The difference in mean phenprocoumon and acenocoumarol dose can be found in -Table 2.

\section{Anticoagulation Control before and after First Vaccination in Subgroups}

In the subgroup of VKA users $(n=2,355)$ who had an INR within range prior to vaccination, $30.8 \%$ had an INR outside their therapeutic range afterwards. In the standard intensity group ( $n=2,170), 329$ (15.2\%) had a subtherapeutic INR $($ INR $<2.0)$ and $330(15.2 \%)$ had a supratherapeutic INR (INR $>3.0)$. In the high-intensity group $(n=185), 36$ $(19.5 \%)$ had a subtherapeutic INR $($ INR $<2.5)$ and 30 (16.2\%) had a supratherapeutic INR (INR $>3.5$ ) after vaccination. The mean INR in VKA users who had an INR within range prior to vaccination was also higher after the first vaccination (mean INR [SD] 2.48 [0.50] vs. 2.55 [0.66], $p<0.001)$. VKA users with a poor TTR $(n=1,041)$ were more likely to have an INR out of range compared with patients with a TTR $>60 \%$ after the first vaccination (653 (31.3\%) vs. 408 (39.2\%), $p=0.001$ ). In patients who had their INR measured within 14 days $(n=2,706)$, similar results as the main analysis were seen.

\section{Anticoagulation Control in Patients Who Completed the Vaccination Program}

The results after the first and second vaccination of the patients who completed the vaccination $(n=1,334)$ are shown in - Tables 3 and $\mathbf{4}$. The percentages of INRs within range after 
Table 1 Clinical characteristics of all vaccine recipients

\begin{tabular}{|c|c|c|}
\hline & Phenprocoumon & Acenocoumarol \\
\hline Patients ( $n, \%)$ & $1,040(33.0)$ & $2,108(67.0)$ \\
\hline Age (SD) & $85.93(9.1)^{b}$ & $87.14(8.4)^{\mathrm{b}}$ \\
\hline Male $(n, \%)$ & $465(44.7)$ & $915(43.4)$ \\
\hline \multicolumn{3}{|l|}{ Treatment indication $^{\mathrm{a}}$} \\
\hline Atrial fibrillation $(n, \%)$ & $828(79.6)$ & $1,700(80.6)$ \\
\hline Venous thrombosis ( $n, \%)$ & $98(9.4)$ & $177(8.4)$ \\
\hline Mechanical heart valves $(n, \%)$ & $37(3.6)$ & $100(4.7)$ \\
\hline Vascular surgery $(n, \%)$ & 19 (1.9) & $35(1.7)$ \\
\hline Ischemic heart disease ( $n, \%)$ & $5(0.5)$ & $13(0.6)$ \\
\hline Other $(n, \%)$ & $53(5.1)$ & $83(3.9)$ \\
\hline \multicolumn{3}{|l|}{ Target INR } \\
\hline$[2.0-3.0],(n, \%)$ & $948(91.2)$ & $1,922(91.2)$ \\
\hline$[2.5-3.5],(n, \%)$ & $92(8.8)$ & $186(8.8)$ \\
\hline
\end{tabular}

Abbreviations: INR, international normalized ratio; SD, standard deviation.

aprimary treatment indication.

${ }^{\mathrm{b}} \mathrm{p}$-Value $<0.001$.

Table 2 anticoagulation levels before and after the first vaccination in every vaccine recipient $(n=3148)$

\begin{tabular}{|l|l|l|}
\hline & Prior vaccination & After first vaccination \\
\hline Standard intensity [2.0-3.0] & & \\
\hline INR below range & $390(13.6 \%)^{\mathrm{b}}$ & $495(17.2 \%)^{\mathrm{b}}$ \\
\hline INR in range & $2,170(75.6 \%)^{\mathrm{b}}$ & $1,914(66.7 \%)^{\mathrm{b}}$ \\
\hline INR above range & $310(10.8 \%)^{\mathrm{b}}$ & $461(16.1 \%)^{\mathrm{b}}$ \\
\hline High intensity [2.5-3.5] & & \\
\hline INR below range & $70(25.2 \%)$ & $66(23.7 \%)$ \\
\hline INR in range & $185(66.5 \%)$ & $166(59.7 \%)$ \\
\hline INR above range & $23(8.3 \%)^{\mathrm{b}}$ & $46(16.5 \%)^{\mathrm{b}}$ \\
\hline INR level (mean, SD) & $2.50(0.57)^{\mathrm{b}}$ & $2.55(0.70)^{\mathrm{b}}$ \\
\hline Phenprocoumon tablets (mean, SD) & $0.49(0.22)^{\mathrm{b}}$ & $0.49(0.22)^{\mathrm{b}}$ \\
\hline Acenocoumarol tablets (mean, SD) & $1.78(0.78)$ & $1.77(0.78)$ \\
\hline INR $\geq 5$ & $14(0.4 \%)$ & $20(0.6 \%)$ \\
\hline Significant dose adjustment & $33(1.0 \%)$ \\
\hline
\end{tabular}

Abbreviations: INR, international normalized ratio; SD, standard deviation.

a Significant dose adjustment is defined as a dose adjustment of $10 \%$ or more.

${ }^{\mathrm{b}} p$-Value $<0.05$, calculated by McNemar's test or paired $t$-tests.

first vaccination and second vaccination were similar. Likewise, no increased risk was observed for reaching an INR below or above range in both groups ( - Table 4 ). The mean INR after the second vaccination was similar to the first vaccination. However, an increase of significant dose adjustments was seen after the second vaccination (13 [1.1\%] vs. 68 [6.0\%], $p<0.001)$. Similar results were seen in VKA users who had an INR in range prior vaccination and in VKA users with an INR measurement of 14 days or shorter after vaccination.

\section{Anticoagulation Control Prior Vaccination Compared with after Second Vaccination}

In the standard intensity group, the percentage of an INR within target range was significantly lower after the second 


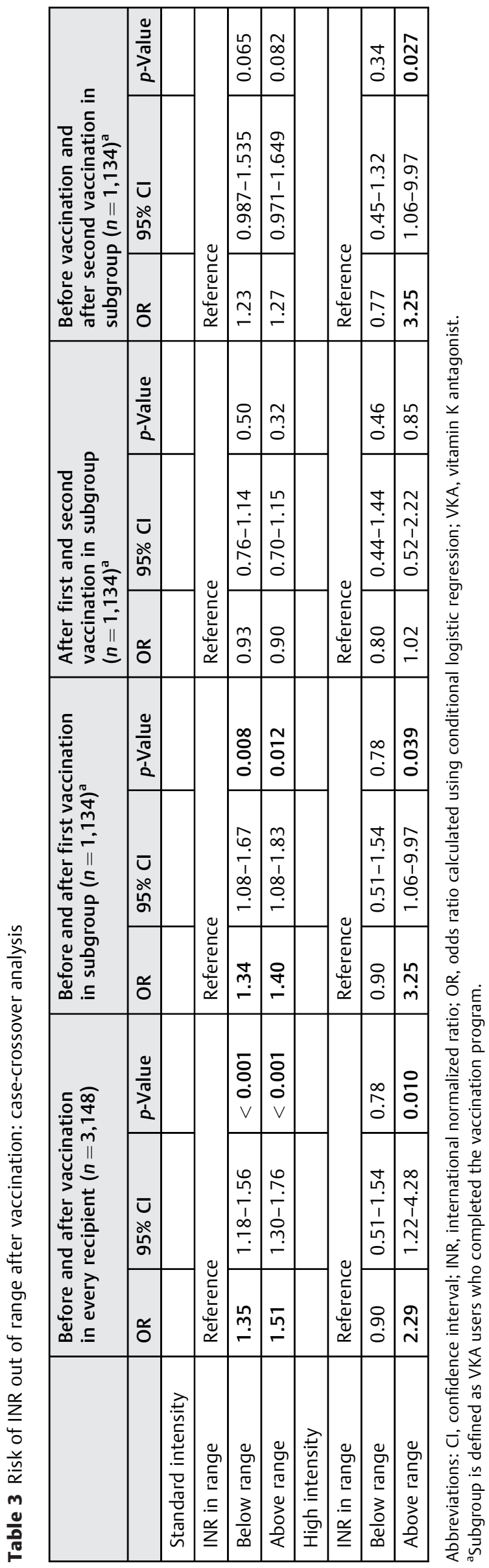

vaccination compared with prior vaccination (753 [73.0\%] vs. 694 [67.3\%], $p=0.004)$. The percentage of subtherapeutic INR (INR < 2.0) was significantly higher after second vaccination than prior vaccination (161 [15.6\%] vs. 195 [18.9\%], $p=0.041)$. In this group, the OR did not differ before and after the vaccination program ( - Table 3 ).

In the high-intensity group, no difference was seen in the percentage of INRs within range after the vaccination program (-Table 4). In the high-intensity group, the risk of supratherapeutic INR levels was 3.25 times higher after completing vaccination compared with prior vaccination (OR 3.25 [95\% CI 1.06-9.97], $p=0.027$ ) (-Table 3). Comparable results were seen in VKA users who had an INR in range prior vaccination and in VKA users with an INR measurement of 14 days or shorter after vaccination.

\section{Sensitivity Analyses}

Sensitivity analyses showed that the percentage of INRs out of range was higher after the first vaccination, irrespective of the chosen baseline INR ( - Supplementary Tables $\mathbf{S 3}$ and $\mathbf{5 4}$, available in the online version). Patients with the most stable INR and standard intensity ( $n=603)$ experienced a decrease from 83.9 to $73.0 \%$ of INRs in range after the first vaccination $(p<0.001)$. The risk of subtherapeutic (OR 1.35 [95\% Cl 1.171.55], $p<0.001$ ) and supratherapeutic INR levels (OR 1.54 [95\% Cl 1.32-1.79], $p<0.001$ ) were both increased after the first vaccination. In the high-intensity group $(n=27)$, we did not observe a difference in the percentage of INRs in range after vaccination. However, the risk of supratherapeutic INR levels was increased after the first vaccination (OR 2.29 [95\% CI 1.22-4.28], $p=0.01$ ).

The mean INR level was significantly higher after the first vaccination, irrespective of intensity. In VKA users who completed the vaccination program, the high-intensity group had a significantly higher INR level after the first and second vaccination $(2.68[0.64]$ vs. 2.99 [0.95], $p=0.007$ and $2.68[0.64]$ vs. 2.92 [0.74], $p=0.01$ ). After we stratified by VKA type, the results followed the main analysis.

\section{Discussion}

Our research aimed to study whether BNT162b2 affects anticoagulation control in outpatients using VKA. Our results indicate that COVID-19 vaccination with BNT162b2 has a significant negative effect on anticoagulation control since $33.3 \%$ of patients had an INR out of range after the first vaccination compared with $24.4 \%$ prior to vaccination. This negative effect was also observed in the most stable VKA users and VKA users who were within range prior to the first vaccination. Nevertheless, BNT162b2 did not result in an increase of the percentage of INR $\geq 5$.

There are several explanations for the effect of vaccination on anticoagulation control. Systemic reactions, including fever and chills, occur in up to $50 \%$ of vaccine recipients receiving the BNT162b2 vaccine. ${ }^{15}$ However, these systemic reactions, are more frequently reported after the second BNT162b2 vaccination. ${ }^{15,24}$ In contrast, the effect of 
Table 4 Anticoagulation levels before and after vaccination in patients who completed the vaccination program $(n=1,134)$

\begin{tabular}{|l|l|l|l|}
\hline & Prior vaccination & After first vaccination & After second vaccination \\
\hline Standard intensity [2.0-3.0], $n=1,031$ & & & \\
\hline Below range & $161(15.6 \%)^{\mathrm{b}, \mathrm{c}}$ & $212(20.6 \%)^{\mathrm{c}}$ & $195(18.9 \%)^{\mathrm{b}}$ \\
\hline INR in range & $753(73.0 \%)^{\mathrm{b}, \mathrm{c}}$ & $667(64.7 \%)^{\mathrm{c}}$ & $694(67.3 \%)^{\mathrm{b}}$ \\
\hline Above range & $117(11.3 \%)^{\mathrm{b}}$ & $152(14.7 \%)^{\mathrm{b}}$ & $142(13.8 \%)$ \\
\hline High intensity [2.5-3.5], $n=103$ & & & \\
\hline Below range & $35(34.0 \%)$ & $28(27.2 \%)$ & $27(26.2 \%)$ \\
\hline INR in range & $60(58.3 \%)$ & $54(52.4 \%)$ & $58(56.3 \%)$ \\
\hline Above range & $8(7.8 \%)^{\mathrm{b}}$ & $21(20.4 \%)^{\mathrm{b}}$ & $18(17.5 \%)$ \\
\hline INR level (mean, SD) & $2.47(0.58)$ & $2.52(0.71)$ & $2.51(0.69)$ \\
\hline Phenprocoumon tablets (mean, SD) & $0.48(0.24)^{\mathrm{b}}$ & $0.48(0.24)$ & $0.48(0.24)^{\mathrm{b}}$ \\
\hline Acenocoumarol tablets (mean, SD) & $1.77(0.84)$ & $1.77(0.84)$ & $1.76(0.84)$ \\
\hline INR $\geq 5$ & $6(0.5 \%)$ & $6(0.5 \%)$ & $8(0.7 \%)$ \\
\hline Significant dose adjustment ${ }^{\mathrm{a}}$ & $11(1.0 \%)$ & $13(1.1 \%)$ & $68(6.0 \%)$ \\
\hline
\end{tabular}

Abbreviations: INR, international normalized ratio; SD, standard deviation.

a Significant dose adjustment is defined as a dose adjustment of $10 \%$ or more.

${ }^{\mathrm{b}} p$-Value $<0.05$.

' $p$-Value $<0.001$ calculated by McNemar's test or paired $t$-tests.

BNT162b2 on anticoagulation control was less pronounced after the second vaccination. This finding, in combination with the increased percentage of dose adjustments, could indicate doctors' anticipation on the effects of vaccination making them dose differently for the second vaccination. Finally, patients themselves could have decided to decrease the dosage in the days following COVID-19 vaccination as they might be afraid for bleeding complications after intramuscular injection. This could result in a higher percentage of subtherapeutic INRs after vaccination.

Another explanation might be the inhibition of cytochrome p-450 caused by vaccination, which is seen in laboratory studies in mice receiving the DTP vaccine. ${ }^{25,26}$ This group of enzymes is responsible for the metabolizing of acenocoumarol and, to a lesser extent, phenprocoumon. ${ }^{27} \mathrm{~A}$ third explanation could be that the modified ribonucleic acid (RNA) encoding the SARS-CoV-2 full-length spike directly affects coagulation. The presence of spike protein S1 can result in structural changes in prohemostatic proteins. ${ }^{28}$ In addition, messenger RNA (mRNA) vaccines can influence coagulation due to excessive extracellular RNA interacting with coagulation factors. ${ }^{29}$

The possible effects of vaccines on anticoagulation control remain debated. Several prospective studies have examined the effect of the influenza vaccine on anticoagulation control. However, their results were conflicting, they had small sample sizes, and none of them were population-based. ${ }^{30}$ Therefore, they might not be generalizable to all adults using VKA. A large retrospective study looked into different vaccines and did not detect any difference of clinical importance in mean INR after vaccination nor any tendency for INR measurements to be outside the therapeutic range. ${ }^{31}$ However, their source data made it impossible to know the therapeutic indication and target range of their study population. The current study provides new insights into this debate by observing the immediate effects of BNT162b2 on INR stability in outpatient VKA users.

Our study has several strengths. First, by using the electronic patient files from four large anticoagulation clinics, we were able to acquire INR results of over 3,000 patients before and after vaccination. This large sample size made it possible to examine subgroups and perform several sensitivity analyses. Second, our study is population-based and thereby giving our results more generalizability than the previously mentioned studies. Collecting INR results both from VKA users who were fully vaccinated and from VKA users who only received the first vaccination minimized selection bias. Interestingly, patients who were fully vaccinated were younger and more often female than those who had received only one vaccination. This is an unusual observation as the Dutch COVID-19 vaccine program invites people first starting with the eldest. One possible explanation is that older people dropped out of the vaccination program more often, which might have been the case if they experienced side effects or complications. Thanks to our study design, we were able to study this phenomenon. Our final strength is that we included acenocoumarol as well as phenprocoumon users. In both users, an immediate effect of BNT162b2 was seen. Compared with the international, frequently used warfarin (half-life 40 hours), acenocoumarol has a relatively short half-life (11 hours) and phenprocoumon a relatively long half-life (140 hours). ${ }^{32}$ As the results were alike in acenocoumarol 
and phenprocoumon users, the negative immediate effect is probably similar for warfarin users.

Our study also has limitations. The first limitation is that we only included patients who received a BNT162b2 vaccine. Still BNT162b2 is the most frequently used vaccine in Europe, with $69 \%$ of the Dutch vaccine recipients vaccinated with BNT162b2. However, caution is needed to generalize our results to the other vaccines, as not every COVID-19 vaccination is an mRNA vaccine. ${ }^{17,33}$ Further research should look into the effects of other vaccine types. Our second limitation is the use of a surrogate variable for bleeding complications, namely an INR $\geq 5$. We could not make any firm conclusions on registered complications, due to the heterogeneity between anticoagulation clinics in registering complications and the low number of complications on the different time points (data not shown). Therefore, we choose an INR $\geq 5$ as surrogate marker. The percentage of INR $\geq 5$ prior to the first vaccination was similar to the percentage after vaccination, suggesting that the risk of bleeding after vaccination is low. Our third limitation is that we cannot exclude the possibility that the negative effect on anticoagulation control was due to dose adjustments to avoid complications. However, vaccination is deemed safe when the INR $<3.5$, so dose adjustment should not have been necessary for vaccination alone. Finally, our study population is older than the average Dutch VKA user, whose mean age is 73 years. ${ }^{34}$ It is unknown whether elderly patients are more prone to be affected by external factors such as vaccines, even though elderly patients usually have a higher TTR than younger patients. ${ }^{23}$ Future studies should also include younger patients to investigate whether the negative effect of COVID-19 vaccination persists in this patient population.

The findings of our study have implications for the management of VKA patients enrolling in a vaccination program. Dutch anticoagulation clinics have been intensely monitoring patients to identify those with INR values over 3.5. In these patients, necessary dose adjustment following INR results took place before vaccination. In patients with an INR within range before vaccination, 30\% were outside range after the first vaccination. Therefore, an INR in range before vaccination is no predictor of INR stability during the vaccination program. Nevertheless, most vaccine recipients stayed in range during the COVID-19 program. Still, even a relatively small effect on anticoagulation control can be meaningful on a population level. Therefore, it is our opinion that frequent INR monitoring shortly after vaccination is advisable.

Although direct oral anticoagulant (DOAC) is the firstline treatment for atrial fibrillation and venous thrombosis, many patients are still treated with VKAs as second-line treatment or because they did not switch to a DOAC. One can postulate that switching VKA to DOAC before COVID-19 vaccination is beneficial for patients without contraindications for DOACs. We think that DOAC users do not experience clinically relevant direct effects of COVID-19 vaccination on the level of anticoagulation, although this has not been studied.

To conclude, our results indicate an immediate negative effect of BNT162b2 on anticoagulation control in patients treated with VKAs. Therefore, it is advisable to monitor the INR shortly after vaccination, even in stable patients.

\section{What is known about this topic?}

- Any systemic event such as illness, fever, or physical stress can decrease anticoagulation control in VKA users.

- Almost 50\% of recipients of COVID-19 vaccine experience systemic effects of the vaccination.

- In patients using vitamin $\mathrm{K}$ antagonists (VKA), the effects of COVID-19 vaccine on the anticoagulation control is uncertain.

\section{What does this paper add?}

- BNT162b2 is associated with an immediate detrimental effect on anticoagulation control in VKA users.

- One-third of VKA users had an INR out of range after the first vaccination compared with $24.4 \%$ prior vaccination.

- This study provides insight into the effects of BNT162b2 on anticoagulation control by using realworld data of more than 3,000 VKA users.

\section{Author Contributions}

C.V., W.M.L., S.C.C., and M.J.H.A.K. participated in the design of the study. C.V. analyzed the data and wrote the manuscript. All authors contributed to the interpretation of the results. J.S.B., M.C.N., F.J.v.d.M., A.J.W.G., Y.C.F. M., S.C.C., W.M.L., and M.J.H.A.K. critically revised the manuscript. All authors read and approved the final manuscript.

\section{Funding}

The Dutch COVID \& Thrombosis Coalition was funded by grants of the Netherlands Thrombosis Foundation (2020_A) and the Netherlands Organization for Health Research and Development (project number 10430012010004).

Conflict of Interest

C.V., J.S.B., M.C.N., F.J..v.d.M., A.J.W.G., Y.C.F.M., S.C.C., and W.M.L. have no conflicts of interest to declare. M.J. H.A.K. has received unrestricted grants paid to the department for research outside this work from Bayer and Daiichi Sankyo, and has received a speaker's fee paid to the department from Bayer.

\section{Acknowledgment}

The authors thank the Netherlands Thrombosis Foundation for making the data available.

\section{References}

1 World Health Organization. Pneumonia of unknown cause China. Disease Outbreak News . January 5, 2020

2 WHO COVID-19 Dashboard. Geneva: World Health Organization. Accessed March 16, 2021: https://covid19.who.int/ 
3 Guan WJ, Ni ZY, Hu Y, et al; China Medical Treatment Expert Group for Covid-19. Clinical characteristics of coronavirus disease 2019 in China. N Engl J Med 2020;382(18):1708-1720

4 Huang C, Wang Y, Li X, et al. Clinical features of patients infected with 2019 novel coronavirus in Wuhan, China. Lancet 2020;395 (10223):497-506

5 Becker RC. COVID-19 update: Covid-19-associated coagulopathy. J Thromb Thrombolysis 2020;50(01):54-67

6 Driggin E, Madhavan MV, Bikdeli B, et al. Cardiovascular considerations for patients, health care workers, and health systems during the COVID-19 pandemic. J Am Coll Cardiol 2020;75(18):2352-2371

7 Tang N, Li D, Wang X, Sun Z. Abnormal coagulation parameters are associated with poor prognosis in patients with novel coronavirus pneumonia. J Thromb Haemost 2020;18(04):844-847

8 Zhou F, Yu T, Du R, et al. Clinical course and risk factors for mortality of adult inpatients with COVID-19 in Wuhan, China: a retrospective cohort study. Lancet 2020;395(10229):1054-1062

9 Klok FA, Kruip MJHA, van der Meer NJM, et al. Incidence of thrombotic complications in critically ill ICU patients with COVID-19. Thromb Res 2020;191:145-147

10 Schein JR, White CM, Nelson WW, Kluger J, Mearns ES, Coleman CI. Vitamin K antagonist use: evidence of the difficulty of achieving and maintaining target INR range and subsequent consequences. Thromb J 2016;14:14

11 Vranckx P, Valgimigli M, Heidbuchel H. The significance of drugdrug and drug-food interactions of oral anticoagulation. Arrhythm Electrophysiol Rev 2018;7(01):55-61

12 Liu S, Li X, Shi Q, et al. Outcomes associated with warfarin time in therapeutic range among US veterans with nonvalvular atrial fibrillation. Curr Med Res Opin 2018;34(03):415-421

13 Ageno W, Gallus AS, Wittkowsky A, Crowther M, Hylek EM, Palareti G. Oral anticoagulant therapy: Antithrombotic Therapy and Prevention of Thrombosis, 9th ed: American College of Chest Physicians Evidence-Based Clinical Practice Guidelines. Chest 2012;141(2, Suppl):e44S-e88S

14 Camilleri E, van Rein N, van der Meer FJM, Nierman MC, Lijfering WM, Cannegieter SCDutch Covid -19 \& Thrombosis Coalition. Stability of vitamin K antagonist anticoagulation after COVID-19 diagnosis. Res Pract Thromb Haemost 2021;5(07):e12597

15 Polack FP, Thomas SJ, Kitchin N, et al; C4591001 Clinical Trial Group. Safety and efficacy of the BNT162b2 mRNA Covid-19 vaccine. N Engl J Med 2020;383(27):2603-2615

16 Baden LR, El Sahly HM, Essink B, et al; COVE Study Group. Efficacy and safety of the mRNA-1273 SARS-CoV-2 vaccine. N Engl J Med 2021;384(05):403-416

17 Voysey M, Clemens SAC, Madhi SA, et al; Oxford COVID Vaccine Trial Group. Safety and efficacy of the ChAdOx1 nCoV-19 vaccine (AZD1222) against SARS-CoV-2: an interim analysis of four randomised controlled trials in Brazil, South Africa, and the UK. Lancet 2021;397(10269):99-111

18 Self TH, Oliphant CS, Reaves AB, Richardson AM, Sands CW. Fever as a risk factor for increased response to vitamin $\mathrm{K}$ antagonists: a review of the evidence and potential mechanisms. Thromb Res 2015;135(01):5-8
19 Federatie van Nederlandse Trombosediensten. Standaard afhandeling VKA-interacties. 2021. Accessed April 1, 2021 at: https:// www.fnt.nl/kwaliteit/VKA-interacties

20 van Rein N, Lijfering WM, Bos MH, et al. Objectives and design of BLEEDS: a cohort study to identify new risk factors and predictors for major bleeding during treatment with vitamin $\mathrm{K}$ antagonists. PLoS One 2016;11(12):e0164485

21 Rosendaal FR, Cannegieter SC, van der Meer FJ, Briët E. A method to determine the optimal intensity of oral anticoagulant therapy. Thromb Haemost 1993;69(03):236-239

22 van Miert JHA, Veeger NJGM, Ten Cate-Hoek AJ, Piersma-Wichers $\mathrm{M}$, Meijer K. Effect of switching from acenocoumarol to phenprocoumon on time in therapeutic range and INR variability: a cohort study. PLoS One 2020;15(07):e0235639

23 Federatie van Nederlandse Trombosediensten. Samenvatting Medische Jaarverslagen 2019. 2019

24 Menni C, Klaser K, May A, et al. Vaccine side-effects and SARS$\mathrm{CoV}-2$ infection after vaccination in users of the COVID Symptom Study app in the UK: a prospective observational study. Lancet Infect Dis 2021;21(07):939-949

25 Ansher SS, Thompson W. Modulation of hepatic mRNA levels after administration of lipopolysaccharide and diphtheria and tetanus toxoids and pertussis vaccine adsorbed (DTP vaccine) to mice. Hepatology 1994;20(4 Pt 1):984-991

26 Ansher S, Thompson W, Bridgewater J, Snoy P. Pertussis toxin-induced alterations of murine hepatic drug metabolism following administration of diphtheria and tetanus toxoids and pertussis vaccine adsorbed. Infect Immun 1993;61(10):4240-4247

27 Ufer M. Comparative pharmacokinetics of vitamin K antagonists: warfarin, phenprocoumon and acenocoumarol. Clin Pharmacokinet 2005;44(12):1227-1246

28 Walsh EE, Frenck RW Jr, Falsey AR, et al. Safety and immunogenicity of two RNA-based Covid-19 vaccine candidates. N Engl J Med 2020;383(25):2439-2450

29 Kannemeier C, Shibamiya A, Nakazawa F, et al. Extracellular RNA constitutes a natural procoagulant cofactor in blood coagulation. Proc Natl Acad Sci U S A 2007;104(15):6388-6393

30 Kuo AM, Brown JN, Clinard V. Effect of influenza vaccination on international normalized ratio during chronic warfarin therapy. J Clin Pharm Ther 2012;37(05):505-509

31 Jackson ML, Nelson JC, Chen RT, Davis RL, Jackson LAVaccine Safety Datalink investigators. Vaccines and changes in coagulation parameters in adults on chronic warfarin therapy: a cohort study. Pharmacoepidemiol Drug Saf 2007;16(07):790-796

32 Hemker HC, Frank HL. The mechanism of action of oral anticoagulants and its consequences for the practice of oral anticoagulation. Haemostasis 1985;15(04):263-270

33 EMA (European Medicines Agency) Summary of opinion: COVID19 Vaccine Janssen,. (COVID-19 vaccine (Ad26.COV2-S [recombinant])). 2021

34 Zielinski GD, van Rein $\mathrm{N}$, Teichert $\mathrm{M}$, et al. Persistence of oral anticoagulant treatment for atrial fibrillation in the Netherlands: a surveillance study. Res Pract Thromb Haemost 2019:4(01):141-153 